\title{
Photoemission studies of yttrium photocathodes by using the visible radiation
}

\author{
J. Scifo $\odot,{ }^{1, *}$ A. Lorusso $\odot,{ }^{2, \dagger}$ E. Chiadroni $\odot,{ }^{1}$ P. Cinquegrana, ${ }^{3}$ S. Dabagov, ${ }^{1}$ M. Danailov, ${ }^{3}$ \\ A. Demidovich $\odot,{ }^{3}$ M. Ferrario, ${ }^{1}$ D. Garzella, ${ }^{4,5}$ A. Giribono, ${ }^{1}$ D. Hampai, ${ }^{1}$ \\ A. Perrone, ${ }^{2}$ and M. Trovò ${ }^{3}$ \\ ${ }^{1}$ Laboratori Nazionali di Frascati, Via Enrico Fermi 54, 00044 Frascati, Italy \\ ${ }^{2}$ Universitá del Salento, Dipartimento di Matematica e Fisica "E. De Giorgi", \\ INFN sezione di Lecce, 73100 Lecce, Italy \\ ${ }^{3}$ Elettra - Sincrotrone Trieste SCpA, S.S. $14 \mathrm{Km} 163.5$ in Area Science Park, \\ 34149 Basovizza - Trieste, Italy \\ ${ }^{4}$ Université Paris-Saclay, CEA, CNRS, LIDYL, 91191, Gif-sur-Yvette, France \\ ${ }^{5}$ Istituto Nazionale di Fisica Nucleare, Piazza dei Caprettari, 70, 00186 Roma, Italy
}

(Received 2 October 2020; accepted 24 November 2020; published 14 December 2020)

\begin{abstract}
The present work reports on Yttrium based photocathodes. A Yttrium (Y) thin film is deposited via pulsed laser deposition (PLD) on the copper $(\mathrm{Cu})$ back flange of a radio frequency (rf) gun for photocathode application. Because of a lower work function with respect to $\mathrm{Cu}, \mathrm{Y}$ photocathodes are particularly appealing for the possibility to illuminate them with visible laser pulses, with the advantage of a higher energy per pulse, paving the way to high repetition rate photoinjectors, driven by conventional laser sources. In addition, working at $\lambda \sim 400 \mathrm{~nm}$ the small energy difference between the $\mathrm{Y}$ work function (about $3 \mathrm{eV}$ ) and the laser photon energy reduces the contribution of the intrinsic emittance of the material. Photoelectrons, emitted by the thin film Y photocathode driven by the second harmonic of a Ti:Sapphire laser, have been characterized in terms of quantum efficiency and transverse emittance. Results have been compared with the theoretical ones obtained by the three-step model of Spicer for metallic photocathodes.
\end{abstract}

DOI: 10.1103/PhysRevAccelBeams.23.123401

\section{INTRODUCTION}

A high peak current and low emittance electron beam is required by several applications in the accelerator physics field, e.g., free electron laser (FEL) radiation sources [1], plasma wake field acceleration (PWFA) experiments [2], generation of $\mathrm{THz}$ radiation $[3,4]$ and inverse Compton scattering sources [5]. This requirement results in a large number of quasimonochromatic electrons, concentrated in very short bunches, with small transverse size and divergence, that is a high particle density $6 \mathrm{D}$ phase space. The $6 \mathrm{D}$ brightness, $\mathrm{B}$, is defined as:

$$
B\left[A / m^{2}\right]=\frac{Q}{\varepsilon_{n x} \varepsilon_{n y} \sigma_{t} \sigma_{\gamma}}
$$

where $\mathrm{Q}$ is the beam charge, $\varepsilon_{n x}$ and $\varepsilon_{n y}$ are respectively the normalized $x x^{\prime}$ and $y y^{\prime}$ transverse emittances, $\sigma_{t}$ is the

\footnotetext{
*Jessica.Scifo@lnf.infn.it

antonella.lorusso@le.infn.it
}

Published by the American Physical Society under the terms of the Creative Commons Attribution 4.0 International license. Further distribution of this work must maintain attribution to the author(s) and the published article's title, journal citation, and DOI. bunch length and $\sigma_{\gamma}$ is the energy spread. The choice and the performance of the photocathode play a fundamental role for the increase of the electron beam final brightness, since the ultimate transverse emittance strongly depends on the electron source. In addition, several applications, in particular high quality gamma ray sources, high energy physics, positrons generation, demand high charge and small emittance electron beams [6-8], therefore, for the above reasons, in the present paper we will concentrate on the quantum efficiency (QE) of the photocathode and on the intrinsic emittance of the photoemitted electrons. In this regard, R\&D activity of the photocathode materials used in photoinjectors is compulsory to improve the quality of electron beams driving new generation of FEL $[9,10]$. Among the metallic photocathodes in use, copper is the most used material due to its chemical resistant against surface degradation as well as for the more relaxed requirements on the vacuum level. However, without appropriate design and treatment [10,11], its QE is relatively low (of the order of $\sim 10^{-5}$ for $\lambda=266 \mathrm{~nm}$ laser driven-photoemission) [11-13] with respect to other metallic photocathodes $\left(3 \times 10^{-4}\right.$ for $\mathrm{Y}$ and $7.6 \times 10^{-4}$ for $\mathrm{Mg}$ at $\lambda=266 \mathrm{~nm}$ ) $[14,15]$. Many solutions have been proposed by the scientific accelerator community to increase the photoemissive properties of photocathodes based on 
copper material. Wang et al. proposed, in 1995, to insert by friction welding process a high $\mathrm{QE}$ material disc $(\mathrm{Mg})$ in a $\mathrm{Cu}$ bulk disc [16], while Qian and collaborators inserted the $\mathrm{Mg}$ disc in a $\mathrm{Cu}$ bulk disc by press fitting process [17]. However, $\mathrm{Mg}$ discs inserted in the $\mathrm{Cu}$ back plate $(10 \mathrm{~cm}$ in diameter and $5 \mathrm{~mm}$ in thickness) of the rf gun cavity have shown problems with rf breakdown at the $\mathrm{Mg}-\mathrm{Cu}$ interface [16]. Metallic thin films deposited directly on the $\mathrm{Cu}$ back plate could avoid such problems. Photocathodes based on metallic thin films deposited by different methods have been prepared by several groups [18-20]. Cultrera et al., in 2007, proposed the pulsed laser deposition (PLD) as a versatile technique for the synthesis of very adherent $\mathrm{Mg}$ thin film for photocathode applications [21,22]. Here after, the PLD has been utilised for the deposition of other metals with interesting claim in the field of photocathode for photoinjectors [23-28]. In particular, $\mathrm{Y}$ is a transition metal with a work function of about $3 \mathrm{eV}$ giving the possibility to drive the photoemission with an incidence radiation in the visible range, i.e., $\lambda \sim 400 \mathrm{~nm}$. This wavelength can be obtained as the second harmonic of Ti:Sa laser, often used in photoinjector facilities, thus avoiding the use of higher harmonic conversions and, with the advantage of an available higher laser energy per pulse and a more stable laser system. In our previous work, $Y$ thin film was deposited on a $\mathrm{Cu}$ polycrystalline substrate for its first testing as a photocathode at the Cavity Test Facility (CTF) of Elettra-Sincrotrone Trieste [29]. The study has been performed using $\lambda=262 \mathrm{~nm}$ laser wavelength showing that the QE of $\mathrm{Y}$ thin film was higher than the $\mathrm{Cu}$ bulk photocathode. The current article presents for the first time the results concerning on the $\mathrm{QE}$ and the total emittance of the electron beam obtained from a Y photocathode based on thin film by using the second harmonic of Ti:Sa. Experimental results have been compared with both theoretical ones, as obtained by the three-step theory [30], and simulated ones, by code ASTRA [31], giving the possibility of deriving for the first time the $\mathrm{Y}$ intrinsic emittance value.

\section{EXPERIMENTAL SETUP}

\section{A. Deposition and characterization of the $Y$ thin film}

The $\mathrm{Y}$ thin film was grown on a $\mathrm{Cu}$ bulk disc of about $10 \mathrm{~cm}$ in diameter in a typical PLD system whose details are described in [29]. The substrate was a polycrystalline $\mathrm{Cu}$ bulk disc of $10 \mathrm{~cm}$ in diameter according to the dimension of the back-flange of the CTF photoinjector. The oxygen-free polycrystalline $\mathrm{Cu}$ substrate surface has been machined by a German company, LT-ULTRA [32], by means of single crystal milling and clean with dry nitrogen. The machining has been done without the use of any oil or cooling fluid (dry machining). Such a process has been defined as $n$-machining [12]. This procedure is useful to reduce roughness and to avoid surface contamination compared to other procedures for example the polishing with diamond paste or the machining with oil. Ex situ characterization of the morphology and the structure of the $\mathrm{Y}$ thin film was performed whose thickness was about $1 \mu \mathrm{m}$ with a diameter of $3 \mathrm{~mm}$. The film was well adherent to the substrate, very uniform with a low droplet density $[25,29]$. The structure and crystal orientation of the film were studied by $\mathrm{x}$-ray diffraction (XRD) measurements performed by a XRD 3003 Seifert $\theta / 2 \theta$ Diffractometer. This instrument is a $2200 \mathrm{~W}$ Power System with a $\mathrm{CuK} \alpha$ anode target, $1 \times 12 \mathrm{~mm}$ beam dimension and angular resolution of $0.001^{\circ}$. The XRD pattern of Fig. 1 (obtained with tension $40 \mathrm{kV}$, current $30 \mathrm{~mA}$, time acquisition $1 \mathrm{sec} /$ step and angular scan $0.02 \%$ step) shows the structure of the Y film which is textured along the (100) plane at $28.23^{\circ}$. The peak at $58.37^{\circ}$ corresponds to $\mathrm{Y}(200)$ plane and the weak peak at $94.04^{\circ} \mathrm{Y}(300)$, being the second and the third orders of interference of Y (100) lattice plane [33]. The other peaks at $43.29^{\circ}, 50.43^{\circ}, 74.13^{\circ}$ and $89.93^{\circ}$ are associated, with $\mathrm{Cu}$ (111), $\mathrm{Cu}$ (100), $\mathrm{Cu}(220)$ and $\mathrm{Cu}(311)$ planes of the $\mathrm{Cu}$ polycrystalline substrate, respectively [33]. After the deposition, the photocathode (on the left of Fig. 2) was placed in a vacuum chamber (on the right of Fig. 2) (0.4 1 volume) filled with Argon at $110 \mathrm{kPa}$ to prevent contamination of the cathode surface during transport to the CTF.

\section{B. Cavity Test Facility (CTF)}

The CTF is located at Elettra-Sincrotrone Trieste. The gun has been installed in the linac tunnel behind the FERMI photoinjector, as is shown in the Fig. 3. The FERMI at Elettra-Sincrotrone Trieste project is a seeded free electron laser (FEL) source, based on the high gain harmonic

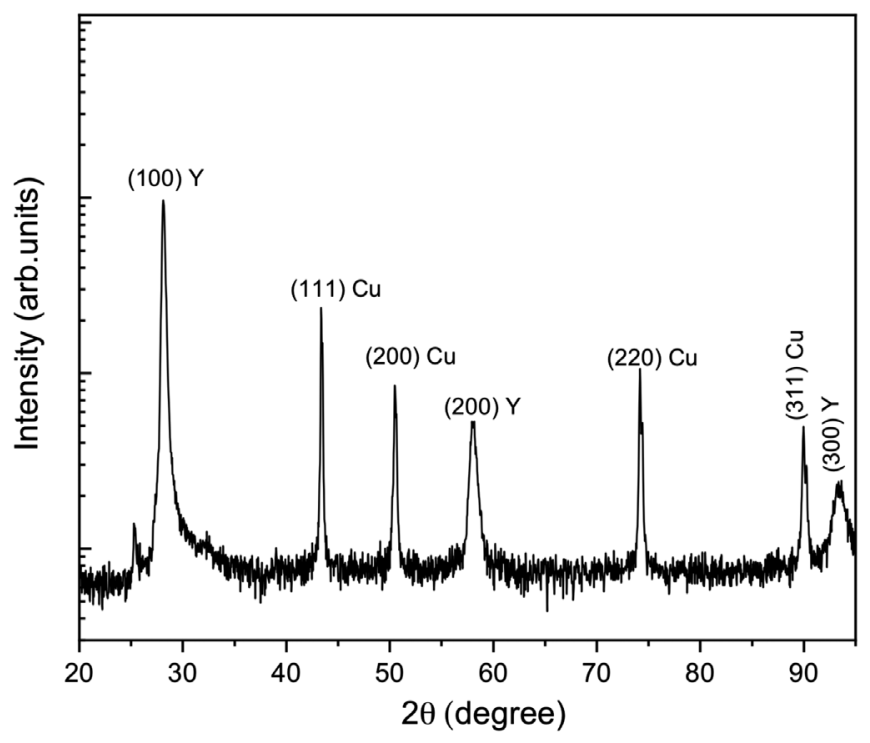

FIG. 1. XRD pattern of the $\mathrm{Y}$ film deposited on the $\mathrm{Cu}$ polycrystalline substrate. 

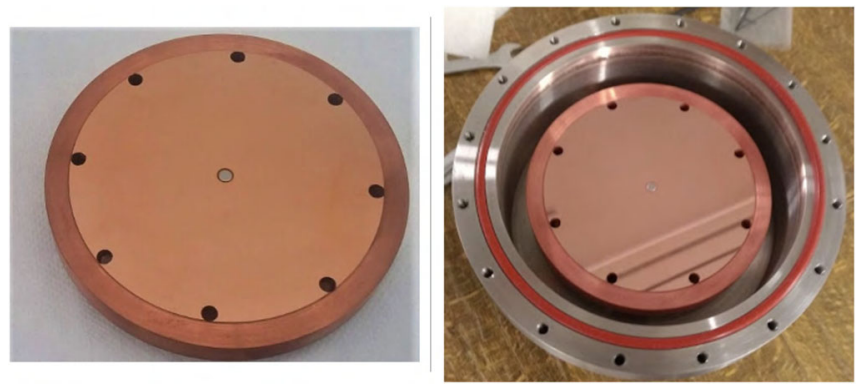

FIG. 2. Left: image of the Y thin film deposited on $\mathrm{Cu}$ center flange. Right: the flange inside the vacuum chamber used for the transport to CTF.

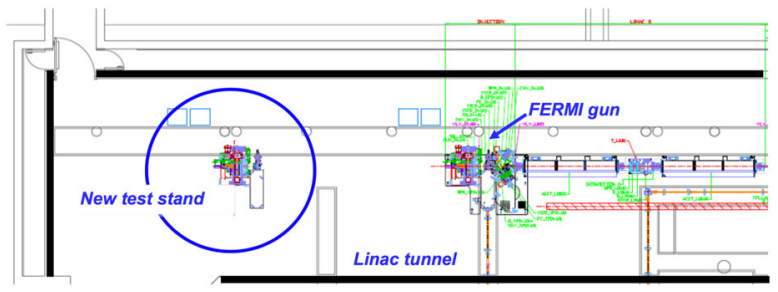

FIG. 3. New test stand in CTF.

generation (HGHG) scheme [34-37]. It is designed to supply photons in a spectral range from 65 to $20 \mathrm{~nm}$ with the first undulator line (FEL-1) and from $20 \mathrm{~nm}$ to $4 \mathrm{~nm}$ with the second undulator line (FEL-2) [38]. The CTF photoinjector consists of a 1.6 cell electron rf gun cavity developed at BNL/SLAC/UCLA operating at the European S-band $(2.998 \mathrm{GHz})$ with an high peak field $(120 \mathrm{MV} / \mathrm{m})$ and powered by a spare klystron. The photocathode is centered on the wall of the flange that terminates the half cell, as shown in Fig. 4.

At the exit of rf gun an emittance compensation solenoid is installed. In Fig. 5 the rf and solenoid field maps of CTF

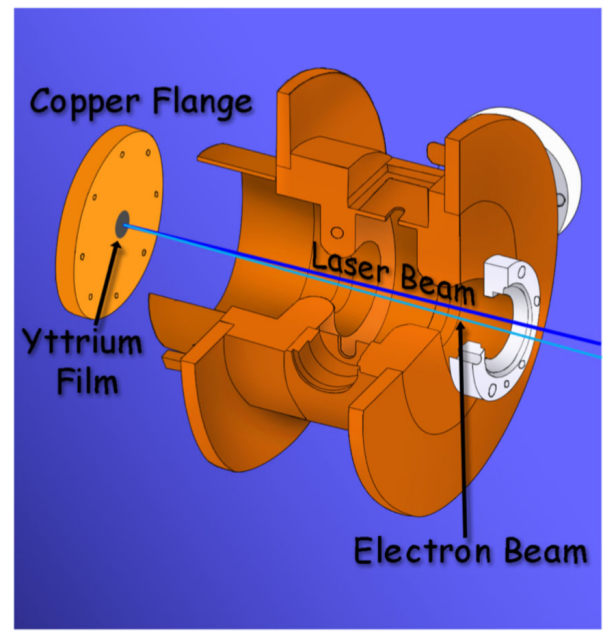

FIG. 4. Drawing of the section of rf gun. The blue line is the laser trajectory, whereas the light blue line is the electron beam.

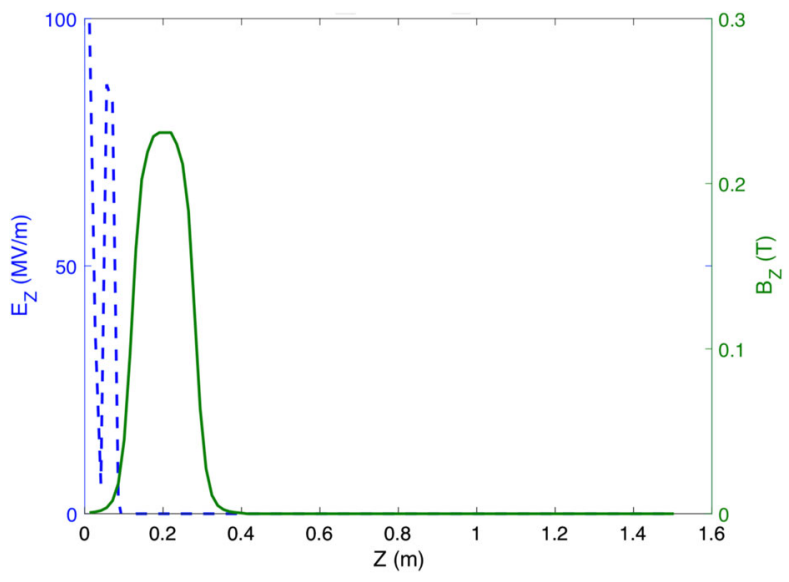

FIG. 5. rf (blue dashed line) and solenoid (green line) fields as seen by the central slice of the electron beam used for the ASTRA simulation. The beam emittance is calculated at the entrance of solenoid, at $Z=0.13 \mathrm{~m}$ where the electron beam has a constant value of Lorentz factor.

beam line are given to ASTRA simulation. A YAG screen is placed downstream from the solenoid, at a distance of $1.177 \mathrm{~m}$ from the photocathode. An integrating current transformer (ICT) is installed before the screen to detect the photoemitted current. Once the electron beam hits the YAG:Ce screen, an optical system directs the emitted light to the CCD camera (Basler SCA 64070), equipped with a Sigma105 macrolens objective, where the beam image is acquired. The FERMI Photoinjector Laser (PIL) is a Ti: Sapphire based laser system containing a regenerative and two two-pass amplifiers delivering 150 fs long infrared pulses with energy of up to $15 \mathrm{~mJ}$. The system has been deliberately designed with excess in IR energy to allow a large margin in the generated UV energy sufficient to compensate the large losses in the pulse and beam shaping systems as well as decrease of the cathode quantum efficiency after prolonged use $[39,40]$. A common-path third-harmonic generation setup consisting of type- 1 BBO crystals is used to generate the $260 \mathrm{~nm}$ range pulse generating the FERMI electron bunch (a bunch charge in the 500-650 pC is typically used depending on the FEL configuration). The unused portion of the second harmonic pulse was extracted through a dichroic mirror and utilized for the CTF facility. The beam transport from the PIL table to the CTF insertion breadboard is made by HR mirrors for all three possible irradiation wavelengths. For the tests described here, only the second harmonic at $392 \mathrm{~nm}$ was transported to the insertion breadboard and then stretched to about $1.5 \mathrm{ps}$ by a pair of high efficiency transmission gratings. The beam size on the cathode was adjusted by the use a fussed silica lens, a fraction of this beam obtained by an uncoated fused silica sampler was used to provide a virtual image for spot size measurement and a real time pulse energy measurement. 


\section{RESULTS AND DISCUSSION}

\section{A. QE measurements}

In the photoemission process, the quantum efficiency, $\mathrm{QE}$, is defined as the ratio of the number of photoemitted electrons $\left(N_{e}\right)$ to the number of incident photons $\left(N_{\phi}\right)$, as expressed in the following equation:

$$
\mathrm{QE}=\frac{N_{e}}{N_{\phi}}=\left(\frac{q}{e}\right) /\left(\frac{E_{L}}{h \nu}\right),
$$

where $q$ is the emitted charge, $e$ is the electron charge, $E_{L}$ is the incident radiation energy on the photocathode, and $h \nu$ is the photon energy.

Table I reports the CTF and laser working parameters used to perform the QE and total beam emittance measurements of $Y$ photocathode. Figure 6 reports the data of the emitted beam charge as a function of the laser energy of the Y photocathode with a linear trend, which indicates the one-photon electron emission. It has to be noticed that during the measurements only the $10 \%$ of the total energy laser available at CTF was sent to the photocathode limiting us in the observation of the space charge effects on the charge emission [41]. The continuous line is the curve fitting. From its slope the $\mathrm{QE}=(1.3 \pm 0.3) \times 10^{-5}$ value is extrapolated, using Eq. (2), and compared with the theoretical one by the Spicer's three-step model [42] as discussed in our previous article [29]. The yttrium optical and physical parameters at $\lambda=392 \mathrm{~nm}$, used to compute the QE, are reported in Table II. $k$ is the complex refraction index of the material, $R$ is the reflectivity of the metal, $\lambda_{\mathrm{ph}}$ is the photon's optical depth, the $\lambda_{e-e}$ is the electron mean free path, $E_{F}$ is the Fermi energy, and $\phi_{\text {work }}$ is the work function. The theoretical QE results to be $1.4 \times 10^{-5}$ which is comparable with the experimental result within the error. Table III highlights the comparable QE values as expected (and measured) for $\mathrm{Y}$ and $\mathrm{Cu}$ photocathodes, with the only difference of the incident laser wavelength. The result is comparable to the experimental data described in [43].

TABLE I. Photoinjector settings and laser parameters for QE and beam emittance measurements.

\begin{tabular}{lc}
\hline \hline Parameters & Value \\
\hline $\mathrm{E}_{\mathrm{rf}}$ & $91 \mathrm{MV} / \mathrm{m}$ \\
Working rf phase & $30^{\circ}$ \\
Energy at the gun exit & $4.15 \mathrm{MeV}$ \\
Laser pulse length & $1.5 \mathrm{ps}-\mathrm{FWHM}(\mathrm{Gaussian})$ \\
Laser wavelength & $\lambda=392 \mathrm{~nm}$ \\
Photon energy & $3.16 \mathrm{eV}$ \\
Laser spot size & $\sigma_{x}=0.19 \pm 0.02 \mathrm{~mm}$, \\
(Gaussian profile) & $\sigma_{y}=0.21 \pm 0.02 \mathrm{~mm}$ \\
\hline \hline
\end{tabular}

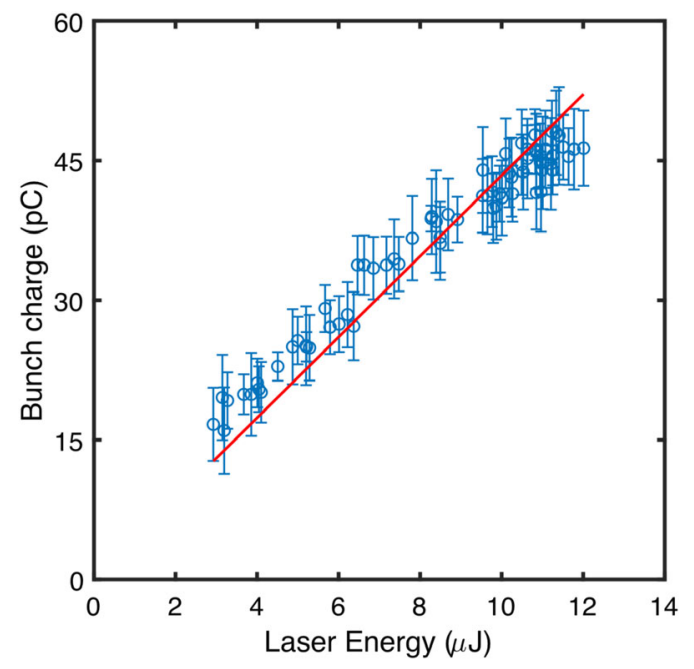

FIG. 6. Collected charge as a function of the laser energy. The solid red line is the linear fit curve. $\mathrm{QE}=(1.3 \pm 0.3) \times 10^{-5}$.

\section{B. Beam emittance measurements as function of bunch charge}

Beam emittance measurements have been performed using the solenoid scan technique for different bunch charges $[44,45]$. The parameters used for the solenoid scan measurements are reported in Table I.

In a solenoid scan beam size measurements for at least three different solenoid settings are required in order to solve for the three independent unknown parameters $\left(\left\langle x_{0^{2}}\right\rangle\right.$, $\left\langle x_{0} x_{0}^{\prime}\right\rangle$, and $\left.\left\langle x_{0}^{\prime 2}\right\rangle\right)$. The experimental layout is indicated in Fig. 7.

Such a system is overdetermined if more than three measurements are done and it can be solved by the standard technique of the $\chi^{2}$ minimization [44]:

TABLE II. Yttrium optical and physical parameters at $\lambda=$ $392 \mathrm{~nm}$ used to compute the QE [25].

\begin{tabular}{lc}
\hline \hline Parameters & Value \\
\hline $\mathrm{k}$ & 0.89 \\
$\mathrm{R}$ & 0.54 \\
$\lambda_{\text {ph }}$ & $154 \AA$ \\
$\lambda_{e-e}$ & $10 \AA$ \\
$E_{F}$ & $6.3 \mathrm{eV}$ \\
$\phi_{\text {work }}$ & $3.1 \mathrm{eV}$ \\
\hline \hline
\end{tabular}

TABLE III. Comparison of $\mathrm{Y}$ an $\mathrm{Cu}$ theoretical $\mathrm{QE}$ value [42] at the same experimental condition reported in Table I.

\begin{tabular}{lccr}
\hline \hline & $\lambda_{\text {laser }}(\mathrm{nm})$ & $\phi_{\text {work }}(\mathrm{eV})$ & \multicolumn{1}{c}{$\mathrm{QE}$} \\
\hline$Y_{\text {experimental }}$ & 392 & 3.1 & $(1.3 \pm 0.3) \times 10^{-5}$ \\
$Y_{\text {theoretical }}$ & 392 & 3.1 & $1.4 \times 10^{-5}$ \\
$\mathrm{Cu}_{\text {theoretical }}$ & 266 & 4.6 & $2.3 \times 10^{-5}$ \\
\hline \hline
\end{tabular}




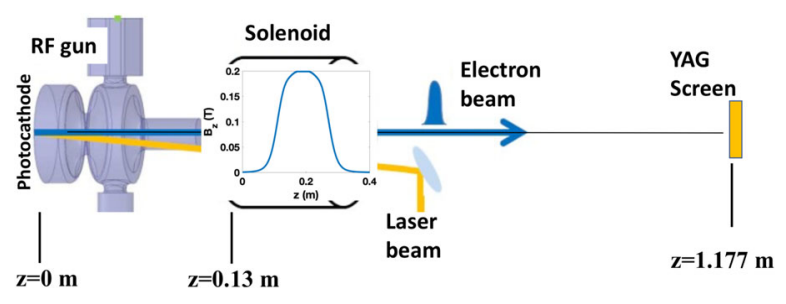

FIG. 7. The solenoid is installed at $z=0.13 \mathrm{~m}$ from the photocathode. The solenoid configuration has the four coils with same fields. After the solenoid and a drift space a YAG:Ce screen is placed perpendicular to the electron beam at $z=1.177 \mathrm{~m}$ (image not in scale).

$$
\left\langle x_{(i)}\right\rangle^{2}=R_{11}^{(i)^{2}}\left\langle x_{0}^{2}\right\rangle+2 R_{11}^{(i)} R_{12}^{(i)}\left\langle x_{0} x_{0}^{\prime}\right\rangle+R_{12}^{(i)^{2}}\left\langle x_{0}^{\prime 2}\right\rangle
$$

where $(i)$ is the number of measurements and the coefficients $R_{11}$ and $R_{12}$ are the elements of the beam line. The normalized emittance formula has been computed at the entrance of the gun solenoid:

$$
\varepsilon_{n x, \mathrm{rms}}=\langle\gamma \beta\rangle \sqrt{\left\langle x_{0}^{2}\right\rangle\left\langle x_{0}^{\prime 2}\right\rangle-\left\langle x_{0} x_{0}^{\prime}\right\rangle^{2}} \text {. }
$$

Measurements of beam size on a YAG:Ce screen, placed $1.177 \mathrm{~m}$ downstream from the solenoid, have been acquired for different solenoid fields. The MATLAB $®$ software environment is used to perform image and data analysis. The images analysis includes subtraction of background (dark current) images and a 95\% charge cut. The results of MATLAB® custom scripts, written for the data analysis, give a plot that represents the data of solenoid scan, the resulting fit (Fig. 8) and the total beam emittance at the solenoid entrance (black dots in Fig. 9 and Fig. 10). The experimental results have been reproduced by means of

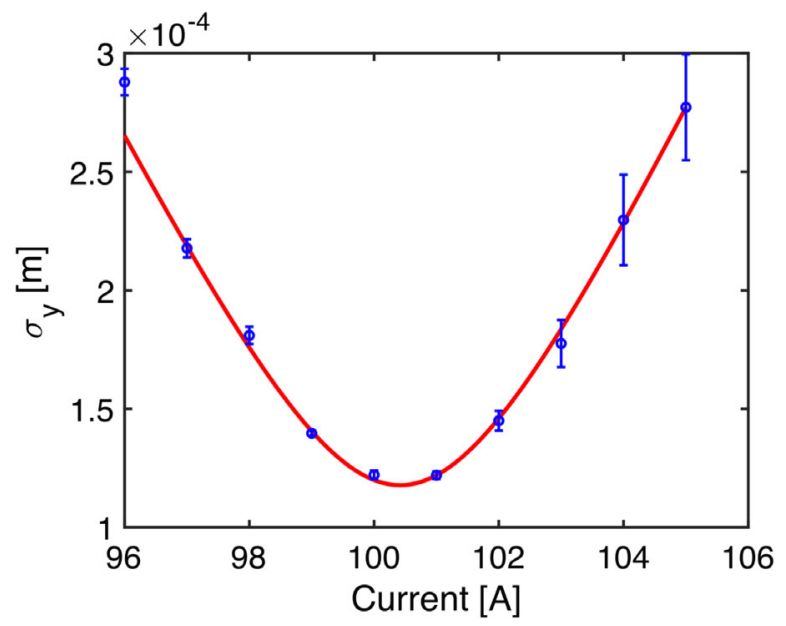

FIG. 8. Typical example of a solenoid scan. The plot shows the beam size versus the solenoid current. Experimental data are reported with blue dots, fit is reported with a solid red line. The bunch charge is $\sim 22 \mathrm{pC}$. Other relevant parameters are reported in Tab. I.

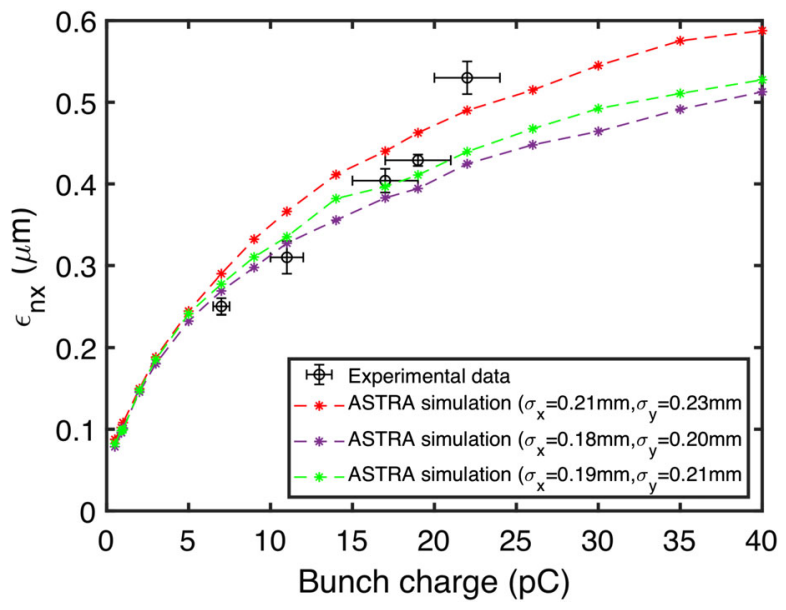

FIG. 9. Total normalized beam emittance as function of bunch charge for $\mathrm{Y}$ thin film on $\mathrm{Cu}$ substrate photocathode (x-plane): measurements (black dots) and ASTRA simulations at different laser spot size (dashed line).

ASTRA simulations for both $\mathrm{x}$ and $\mathrm{y}$ plane, respectively Fig. 9 and Fig. 10. ASTRA is a multi-particle code that includes space charge and intrinsic emittance calculations. Moreover, by using ASTRA is possible to model the photoemission process from a metallic cathode at room temperature when a Fermi-Dirac beam distribution impinges on the cathode.

An extensive simulation campaign has been performed to take into account as much as possible the uncertain on the laser parameter measurements. In Fig. 9 and Fig. 10 is reported the behaviour of the horizontal and vertical beam emittance versus its charge for three spot size configurations, each representing the best (violet line) and worst (red line) case scenario if one considers as extremes for the laser spot size the ones reported in Table I. A good agreement

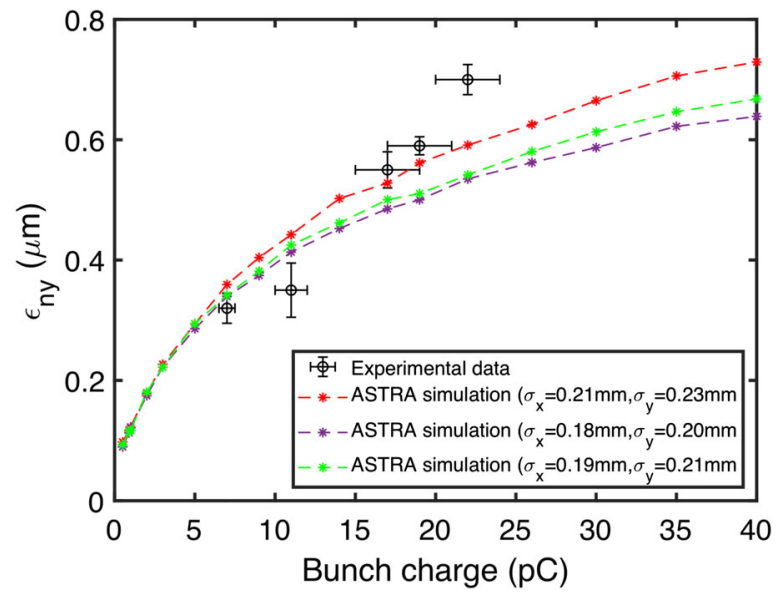

FIG. 10. Total normalized beam emittance as function of bunch charge for $\mathrm{Y}$ thin film on $\mathrm{Cu}$ substrate photocathode (y-plane): measurements (black dots) and ASTRA simulations at different laser spot size (dashed line). 
TABLE IV. Comparison of experimental and theoretical Y and theoretical $\mathrm{Cu}$ intrinsic emittance value [Eq. (5)] at the same experimental condition reported in Table I.

\begin{tabular}{lccc}
\hline \hline & $\lambda_{\text {laser }}(\mathrm{nm})$ & $\phi_{\text {work }}(\mathrm{eV})$ & $\varepsilon_{n, \text { int }} / \mathrm{mm}(\mu \mathrm{m} / \mathrm{mm})$ \\
\hline$Y_{\text {experimental }}$ & 392 & 3.1 & $0.46 \pm 0.02$ \\
$Y_{\text {theoretical }}$ & 392 & 3.1 & 0.45 \\
$\mathrm{Cu}_{\text {theoretical }}$ & 266 & 4.6 & 0.52 \\
\hline \hline
\end{tabular}

between measurements and simulations was achieved considering a $95 \%$ emittance.

The intrinsic emittance value has been extrapolated from the measurements with a cross-check procedure using ASTRA simulations. The theoretical intrinsic emittance value is given by the following formula [Eq. (5)] [46]:

$$
\varepsilon_{n, \mathrm{int}}=\sigma_{x} \sigma_{p_{x}}=\sigma_{x} \sqrt{\frac{\hbar \omega-\phi_{\mathrm{eff}}}{3 m c^{2}}} .
$$

where $\sigma_{x}$ is the laser spot size, $\hbar \omega$ is the photon energy, $\phi_{\text {eff }}$ $(2.84 \mathrm{eV})$ is the effective work function defined as the difference between the work function for the emission, $\phi_{\text {work }}$, and the $\phi_{\text {Schottky }}$ that is the reduction in the potential barrier due to the external applied field, $E_{r f}, m$ is the electron mass and $c$ is the speed of light. The intrinsic emittance value has been extrapolated from the ASTRA simulation turning off the space charge field at beam charge near to zero $(Q \sim 0 \mathrm{pC})$. In this way we are confident that the total beam normalized emittance near the cathode, at $z=0.008 \mathrm{~m}$, is only due to the intrinsic emittance. In this condition the $100 \%$ emittance results to be $\varepsilon_{n x \text {,int }}=$ $0.094 \mu \mathrm{m}$ and $\varepsilon_{n y, \text { int }}=0.108 \mu \mathrm{m}$ in the worst case scenario and $\varepsilon_{n x, \text { int }}=0.083 \mu \mathrm{m}$ and $\varepsilon_{n y, \text { int }}=0.092 \mu \mathrm{m}$ in the best case scenario. These values turn in an intrinsic emittance per unit laser spot size of $\varepsilon_{n \text {,int }} / \mathrm{mm}=0.46 \pm 0.02 \mu \mathrm{m} / \mathrm{mm}$. This value is in good agreement with the intrinsic emittance $\varepsilon_{\text {theoretical }, n \text {,int }} / \mathrm{mm}=0.45 \mu \mathrm{m} / \mathrm{mm}$ calculated from the theoretical equation [Eq. (5)], with residual discrepancies related to uncertain on the laser beam parameters as suggested in Fig. 9 and Fig. 10.

Following a summarizing table (Tab. IV) shows the experimental and theoretical intrinsic emittance value per unit laser spot size for the yttrium and the comparison with respect to the theoretical value for the copper, at the standard UV wavelength, $\lambda=266 \mathrm{~nm}$, using the experimental parameters reported in Table I.

Also for the intrinsic emittance value, the result is comparible to the experimental data reported in [43].

\section{CONCLUSIONS}

First detailed photoemission studies of $\mathrm{Y}$ thin film on $\mathrm{Cu}$ photo-cathodes using visible incident radiation have been presented. QE and normalized beam emittance measurements have been performed using the solenoid scan technique for different bunch charges. The QE measurement of the $\mathrm{Y}$ thin film on $\mathrm{Cu}$ photocathode showed that the experimental value is $\mathrm{QE}=(1.3 \pm 0.3) \times 10^{-5}$. This value is comparable with the theoretical value that is $\mathrm{QE}=1.4 \times 10^{-5}$, computed by the three-step model of Spicer. The experimental data follow a linear trend, which indicates the one-photon electron emission. The space charge effects are not visible.

Regarding the beam emittance measurements the experimental data show a good agreement with ASTRA simulations data. Indeed, the intrinsic emittance per unit laser beam size is $\varepsilon_{n, \text { int }} / \mathrm{mm}=0.46 \pm 0.02 \mu \mathrm{m} / \mathrm{mm}$, that is close to the intrinsic emittance calculated from the theoretical equation [Eq. (5)], that is $\varepsilon_{\text {theoretical, } n \text {,int }} / \mathrm{mm}=$ $0.45 \mu \mathrm{m} / \mathrm{mm}$. The results obtained in this work are very promising for using $\mathrm{Y}$ as photocathode instead of the conventional $\mathrm{Cu}$ which is the most commonly used metals in the rf gun. Although the QE and the emittance values of $\mathrm{Y}$ are comparable with that ones of $\mathrm{Cu}$, the possibility to drive the photoemission with a radiation in the visible range avoids the conversion to higher laser harmonics. This solution increases the laser energy per pulse, getting electron bunches with more available charge.

[1] M. Cornacchia, Lcls x-ray fel at slac, in Free-Electron Laser Challenges II, Vol. 3614 (International Society for Optics and Photonics, San Jose, CA, 1999), pp. 109-118.

[2] M. Litos, E. Adli, W. An, C. Clarke, C. Clayton, S. Corde, J. Delahaye, R. England, A. Fisher, J. Frederico et al., High-efficiency acceleration of an electron beam in a plasma wakefield accelerator, Nature (London) 515, 92 (2014).

[3] F. Giorgianni, E. Chiadroni, A. Rovere, M. Cestelli-Guidi, A. Perucchi, M. Bellaveglia, M. Castellano, D. Di Giovenale, G. Di Pirro, M. Ferrario et al., Strong nonlinear terahertz response induced by dirac surface states in bi 2 se 3 topological insulator, Nat. Commun. 7, 11421 (2016).

[4] E. A. Nanni, W. R. Huang, K.-H. Hong, K. Ravi, A. Fallahi, G. Moriena, R. D. Miller, and F. X. Kärtner, Terahertz-driven linear electron acceleration, Nat. Commun. 6, 8486 (2015).

[5] A. Bacci, D. Alesini, P. Antici, M. Bellaveglia, R. Boni, E. Chiadroni, A. Cianchi, C. Curatolo, G. Di Pirro, A. Esposito et al., Electron linac design to drive bright compton back-scattering gamma-ray sources, J. Appl. Phys. 113, 194508 (2013).

[6] V. Petrillo, A. Bacci, R. B. A. Zinati, I. Chaikovska, C. Curatolo, M. Ferrario, C. Maroli, C. Ronsivalle, A. Rossi, L. Serafini et al., Photon flux and spectrum of $\gamma$-rays compton sources, Nucl. Instrum. Methods Phys. Res., Sect. A 693, 109 (2012).

[7] Y. Jiao, X. Cui, Z. Duan, Y. Guo, P. He, X. Huang, D. Ji, H. Ji, C. Li, J. Li et al., Progress of lattice design and physics studies on the high energy photon source, in 9th Int. Particle Accelerator Conf.(IPAC'18), Vancouver, Canada (JACoW, Geneva, 2018). 
[8] N. Oshima, R. Suzuki, T. Ohdaira, A. Kinomura, T. Narumi, A. Uedono, and M. Fujinami, Brightness enhancement method for a high-intensity positron beam produced by an electron accelerator, J. Appl. Phys. 103, 094916 (2008).

[9] D. Dowell, I. Bazarov, B. Dunham, K. Harkay, C. HernandezGarcia, R. Legg, H. Padmore, T. Rao, J. Smedley, and W. Wan, Cathode r\&d for future light sources, Nucl. Instrum. Methods Phys. Res., Sect. A 622, 685 (2010).

[10] F. Le Pimpec, C. Milne, C. Hauri, and F. Ardana-Lamas, Quantum efficiency of technical metal photocathodes under laser irradiation of various wavelengths, Appl. Phys. A 112, 647 (2013).

[11] F. Zhou, A. Brachmann, F. Decker, P. Emma, S. Gilevich, R. Iverson, P. Stefan, and J. Turner, High-brightness electron beam evolution following laser-based cleaning of a photocathode, Phys. Rev. Accel. Beams 15, 090703 (2012).

[12] J. Scifo, D. Alesini, M. Anania, M. Bellaveglia, S. Bellucci, A. Biagioni, F. Bisesto, F. Cardelli, E. Chiadroni, A. Cianchi et al., Nano-machining, surface analysis and emittance measurements of a copper photocathode at sparc_lab, Nucl. Instrum. Methods Phys. Res., Sect. A 909, 233 (2018).

[13] J. R. Maldonado, Z. Liu, D. Dowell, R. E. Kirby, Y. Sun, P. Pianetta, and F. Pease, Robust csbr/cu photocathodes for the linac coherent light source, Phys. Rev. Accel. Beams 11, 060702 (2008).

[14] A. Lorusso, F. Gontad, L. Solombrino, E. Chiadroni, E. Broitman, and A. Perrone, Tight comparison of $\mathrm{mg}$ and $\mathrm{y}$ thin film photocathodes obtained by the pulsed laser deposition technique, Nucl. Instrum. Methods Phys. Res., Sect. A 836, 57 (2016).

[15] F. Le Pimpec, C. Gough, M. Paraliev, R. Ganter, C. Hauri, and S. Ivkovic, Vacuum breakdown limit and quantum efficiency obtained for various technical metals using dc and pulsed voltage sources, J. Vacuum Sci. Technol. A 28, 1191 (2010).

[16] X. Wang, T. S. Rao, K. Batchelor, I. Ben-Zvi, and J. Fischer, Measurements on photoelectrons from a magnesium cathode in a microwave electron gun, Nucl. Instrum. Methods Phys. Res., Sect. A 356, 159 (1995).

[17] H. Qian, J. Murphy, Y. Shen, C. Tang, and X. Wang, Surface photoemission in a high-brightness electron beam radio frequency gun, Appl. Phys. Lett. 97, 253504 (2010).

[18] T. Srinivasan-Rao, J. Fischer, and T. Tsang, Photoemission studies on metals using picosecond ultraviolet laser pulses, J. Appl. Phys. 69, 3291 (1991).

[19] T. Srinivasan-Rao, J. Schill, I. B. Zvi, and M. Woodle, Sputtered magnesium as a photocathode material for $\mathrm{rf}$ injectors, Rev. Sci. Instrum. 69, 2292 (1998).

[20] J. Lorkiewicz, R. Nietubyć, R. Diduszko, J. Sekutowicz, A. Kosińska, R. Mirowski, M. Kuk, and A. Trembicki, Coating in ultra-high vacuum cathodic-arc and processing of $\mathrm{pb}$ films on nb substrate as steps in preparation of nb-pb photocathodes for radio-frequency, superconducting e-guns, Vacuum 179, 109524 (2020).

[21] L. Cultrera, G. Gatti, F. Tazzioli, A. Perrone, P. Miglietta, C. Ristoscu, S. Orlanducci, and A. Fiori, Mg based photocathodes for high brightness rf photoinjectors, Appl. Surf. Sci. 253, 6531 (2007).
[22] L. Cultrera, G. Gatti, P. Miglietta, F. Tazzioli, A. Perrone, J. Moody, and P. Musumeci, Electron emission characterization of mg photocathode grown by pulsed laser deposition within an s-band rf gun, Phys. Rev. Accel. Beams 12, 043502 (2009).

[23] L. Cultrera, S. Grigorescu, G. Gatti, P. Miglietta, F. Tazzioli, and A. Perrone, Photoelectron emission from yttrium thin films prepared by pulsed laser deposition, J. Nanosci. Nanotechnol. 9, 1585 (2009).

[24] A. Lorusso, Overview and development of metallic photocathodes prepared by laser ablation, Appl. Phys. A 110, 869 (2013).

[25] A. Lorusso, M. Anni, A. Caricato, F. Gontad, A. Perulli, A. Taurino, A. Perrone, and E. Chiadroni, Deposition of y thin films by nanosecond uv pulsed laser ablation for photocathode application, Thin Solid Films 603, 441 (2016).

[26] A. Lorusso, A. Perrone, and F. Gontad, Overview on development of metallic and superconducting photocathodes by the PLD technique for linear accelerator sources, Nucl. Instrum. Methods Phys. Res., Sect. A 942, 162429 (2019).

[27] L. Cultrera, G. Gatti, and A. Lorusso, Photoemission studies on yttrium thin films, Radiat. Eff. Defects Solids 165, 609 (2010).

[28] A. Lorusso, L. Cultrera, V. Fasano, and A. Perrone, Detailed studies of photocathodes based on $\mathrm{Y}$ thin films grown by PLD technique, Nucl. Instrum. Methods Phys. Res., Sect. B 269, 3091 (2011).

[29] A. Lorusso, M. Trovò, A. Demidovich, P. Cinquegrana, F. Gontad, E. Broitman, E. Chiadroni, and A. Perrone, Pulsed laser deposition of yttrium photocathode suitable for use in radio-frequency guns, Appl. Phys. A 123, 779 (2017).

[30] D. H. Dowell and J. F. Schmerge, Quantum efficiency and thermal emittance of metal photocathodes, Phys. Rev. Accel. Beams 12, 074201 (2009).

[31] K. Flöttmann, Information on http://www.desy.de/mpyflo (2016).

[32] "www.lt-ultra.de".

[33] A. Lorusso, F. Gontad, A. Caricato, E. Chiadroni, E. Broitman, and A. Perrone, Structural and morphological properties of metallic thin films grown by pulsed laser deposition for photocathode application, Appl. Phys. A 122, 162 (2016).

[34] M. Labat, M. Bellaveglia, M. Bougeard, B. Carré, F. Ciocci, E. Chiadroni, A. Cianchi, M. Couprie, L. Cultrera, M. Del Franco et al., High-Gain Harmonic-Generation Freeelectron Laser Seeded by Harmonics Generated in Gas, Phys. Rev. Lett. 107, 224801 (2011).

[35] L. Giannessi, M. Bellaveglia, E. Chiadroni, A. Cianchi, M. Couprie, M. Del Franco, G. Di Pirro, M. Ferrario, G. Gatti, M. Labat et al., Superradiant Cascade in a Seeded Free-Electron Laser, Phys. Rev. Lett. 110, 044801 (2013).

[36] E. Allaria, L. Badano, S. Bassanese, F. Capotondi, D. Castronovo, P. Cinquegrana, M. Danailov, G. D'Auria, A. Demidovich, R. De Monte et al., The fermi freeelectron lasers, J. Synchrotron Radiat. 22, 485 (2015).

[37] G. Penco, E. Allaria, L. Badano, P. Cinquegrana, P. Craievich, M. Danailov, A. Demidovich, R. Ivanov, A. Lutman, L. Rumiz et al., Optimization of a high brightness 
photoinjector for a seeded fel facility, J. Instrum. 8, P05015 (2013).

[38] C. Bocchetta, A. Abrami, E. Allaria, I. Andrian, D. Bacescu, L. Badano, L. Banchi, D. Bulfone, C. Bontoiu, R. Bracco, F. Cargnello, K. Casarin, M. Cornacchia, P. Craievich, D. Cocco, M. Danailov, G. D'Auria, A. Demidovich, G. Ninno, and D. Zangrando, FERMI@Elettra Conceptual Design Report (2007).

[39] M. Danailov, A. Demidovich, R. Ivanov, I. Nikolov, and P. Sigalotti, Performance of the fermi fel photoinjector laser, in Proceedings of the 29th Free Electron Laser Conference, Novosibirsk, Russia (BINP, Novosibirsk, 2007), pp. 358-361.

[40] M. Danailov, Y. Loiko, A. Demidovich, I. Nikolov, P. Sigalotti, and R. Ivanov, Laser systems for next generation light sources, in Proceedings of the 23rd Particle Accelerator Conference, Vancouver, Canada, 2009 (IEEE, Piscataway, NJ, 2009).

[41] P. Musumeci, L. Cultrera, M. Ferrario, D. Filippetto, G. Gatti, M. Gutierrez, J. Moody, N. Moore, J. Rosenzweig, C. Scoby et al., Multiphoton photoemission from a copper cathode illuminated by ultrashort laser pulses in an $\mathrm{rf}$ photoinjector, Phys. Rev. Lett. 104, 084801 (2010).
[42] W. E. Spicer and A. Herrera-Gomez, Modern theory and applications of photocathodes, in Photodetectors and Power Meters, Vol. 2022 (International Society for Optics and Photonics, San Diego, CA, 1993), pp. 18-35.

[43] C. Hauri, R. Ganter, F. Le Pimpec, A. Trisorio, C. Ruchert, and $\mathrm{H}$. Braun, Intrinsic Emittance Reduction of an Electron Beam from Metal Photocathodes, Phys. Rev. Lett. 104, 234802 (2010).

[44] A. Mostacci, M. Bellaveglia, E. Chiadroni, A. Cianchi, M. Ferrario, D. Filippetto, G. Gatti, and C. Ronsivalle, Chromatic effects in quadrupole scan emittance measurements, Phys. Rev. Accel. Beams 15, 082802 (2012).

[45] W. Graves, L. DiMauro, R. Heese, E. Johnson, J. Rose, J. Rudati, T. Shaftan, B. Sheehy, L.-H. Yu, and D. Dowell, Duvfel photoinjector dynamics: Measurement and, simulation, in Proceedings of the 2001 Particle Accelerator Conference, 2001 (IEEE, New York, 2001), Vol. 3, pp. 2230-2232.

[46] W. Graves, L. DiMauro, R. Heese, E. Johnson, J. Rose, J. Rudati, T. Shaftan, and B. Sheehy, Measurement of thermal emittance for a copper photocathode, in Proceedings of the 2001 Particle Accelerator Conference, 2001 (IEEE, New York, 2001), Vol. 3, pp. 2227-2229. 